\title{
Author Correction: piRNA-independent function of PIWIL1 as a co-activator for anaphase promoting complex/cyclosome to drive pancreatic cancer metastasis
}

Feng Li, Peng Yuan, Ming Rao, Chun-Hui Jin, Wei Tang, Ye-Fei Rong, Yun-Ping Hu, Fengjuan Zhang, Tao Wei, Qi Yin, Tingbo Liang, Ligang Wu, Jinsong Li(D), Dangsheng Li, Yingbin Liu, Wenhui Lou, Shuang Zhao (D) and Mo-Fang Liu (D)

Correction to: Nature Cell Biology https://doi.org/10.1038/s41556-020-0486-z, published online 16 March 2020.

In the version of this article originally published, the authors inadvertently used a source image of the "PIWIL1" panel in Fig. 4e as an image for the "Mock" panel in Fig. 6e. The image of "Mock" panel in Fig. 6e has now been replaced with the correct one. Additionally, the source data for the full dataset of Figs. 4e and 6e have been deposited to Figshare (https://doi.org/10.6084/m9.figshare.12032724.v2), and the figure legends have been amended to reflect this in Figs. 4 and 6, respectively. The errors have been corrected in the HTML and PDF versions of the paper.
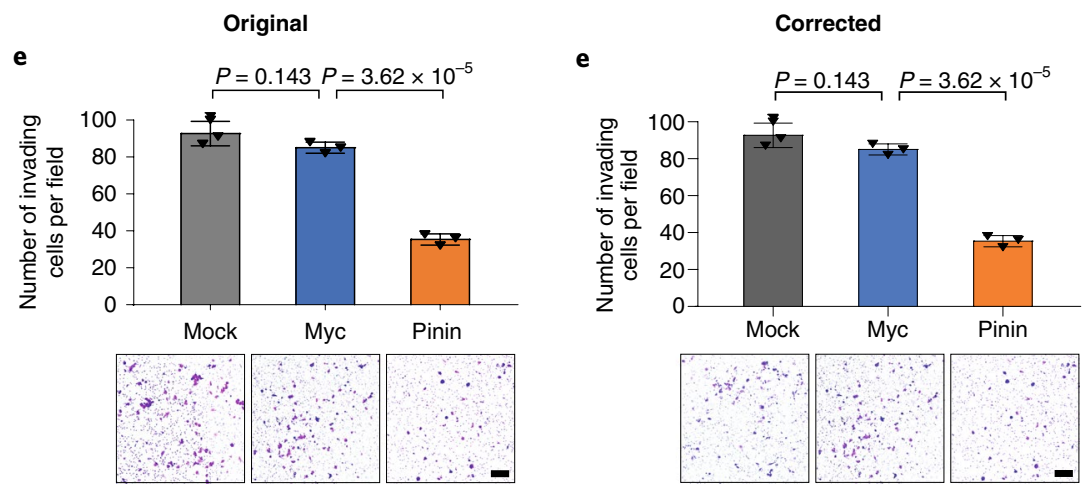

Fig. 6 | Original and Corrected.

Published online: 15 April 2020

https://doi.org/10.1038/s41556-020-0520-1

(๑) The Author(s), under exclusive licence to Springer Nature Limited 2020 